\title{
Transformation of the BeefSpecs fat calculator: Addressing eating quality and production efficiency with on-farm decision making
}

\author{
B.J. Walmsley $^{\text {a,b }}{ }^{\text {, V.H. Oddy }}{ }^{\text {b }}$, B.W. Gudex ${ }^{\text {b }}$, D.G. Mayer ${ }^{\mathrm{c}}$ and M.J. McPhee $^{\mathrm{b}}$ \\ ${ }^{a}$ Animal Breeding and Genetics Unit, ${ }^{b}$ Beef Industry Centre, New South Wales Department of Primary \\ Industries, University of New England, Armidale, New South Wales, ${ }^{c}$ DAF, Dutton Park, Queensland. \\ Email: brad.walmsley@industry.nsw.gov.au
}

\begin{abstract}
The BeefSpecs fat calculator is a decision support tool conceived to assist beef producers with their decision making to achieve better compliance with domestic and international market specifications. BeefSpecs combines data obtained from beef cattle growth-path studies and the extensive body of knowledge contained in animal growth and body composition models with easy to record on-farm measurements to make real-time predictions of body composition. To facilitate producer acceptance and uptake, BeefSpecs makes explicit use of practical end-user knowledge, captured by the simple user interface, by translating it for incorporation into the underpinning research models and returning the outputs in producer language that is easily locatable on the interface. The current version of BeefSpecs (version 1) has three functional forms:

- The primary interface acts as an educational tool to demonstrate the relationship(s) between management decisions and the performance of animal groups,

- The second interface is designed to facilitate animal management on-farm by assisting drafting decisions for creating sub-groups based on predicted performance, and

- The final interface optimises feeding and marketing decisions to increase profitability in both feedlots and pasture finishing systems.
\end{abstract}

The BeefSpecs calculator currently addresses consumer concerns surrounding portion size and levels of subcutaneous fat deposition by focusing on hot standard carcass weight (HSCW; kg) and carcass P8 rump fat depth (P8 fat; $\mathrm{mm}$ ) specifications. However, other carcass attributes influence consumer perceptions of meat quality and production efficiency. Intramuscular fat content, or marbling, has been shown to have positive effects on consumer eating experiences while not receiving the negative perceptions associated with high levels of subcutaneous fat. Carcass yield, as described by lean meat yield, is associated with increased efficiency at the abattoir and remainder of the beef supply chain. These efficiency improvements are reflected in higher premiums reported by the National Livestock Reporting Scheme for higher muscled, higher yielding animals. These attributes have also been combined with other production variables to create a prediction of overall meat quality in a system known as the Meat Standards Australia index, or MSA index.

The evolution of BeefSpecs to improve compliance and the viability of beef production needs to mirror the continued evolution of market specifications to address changing consumer demands. Currently, the Meat Animal Research Centre (MARC) model underlying BeefSpecs predicts composition of empty body weight using a description of animal type and growth rate $(\mathrm{kg} /$ day). Current BeefSpecs inputs and the MARC model are built upon by partitioning lean and fat in the empty body into carcass and non-carcass components with fat being further partitioned into carcass fat depots allowing carcass lean and intramuscular fat to be used to predict marble score. An additional input, muscle score, is used to scale components of the MARC model to make predictions of carcass fatness and lean meat yield. In order to combine the predictions of marble score and lean meat yield with rib fat and HSCW predictions to make a prediction of eating quality using the MSA index, a prediction of ossification score was developed using current BeefSpecs inputs. These enhancements are all designed to improve the utility of all three interface versions of BeefSpecs, with muscle score being the only additional input required. These enhancements also boost the compatibility BeefSpecs has with the national carcass feedback mechanism, Livestock Data Link (LDL), which will allow the impacts that management decisions have on a wider range of carcass traits to be explored with greater emphasis on consumer requirements.

Keywords: $\quad$ Decision Support Systems (DSS), beef cattle, model development, market specifications 


\section{INTRODUCTION}

Management decisions are continually made by beef producers that impact on the capacity of their cattle to meet market specifications and ultimately the profitability of their beef businesses. Analysis of carcass performance in recent years for feedlot and pasture-fed cattle has demonstrated non-compliance to market specifications is high in the Australian beef industry (Slack-Smith et al., 2009, McPhee and Walmsley, 2014). In more detail an analysis of 20,000 records from short-fed feedlot cattle and 32,000 records from pasture-fed cattle indicated that between $15 \%$ and $28 \%$ of carcasses missed hot standard carcass weight (HSCW, $\mathrm{kg}$ ) specifications while between $10 \%$ and $20 \%$ of carcasses missed fat depth $(\mathrm{mm})$ specifications. These noncompliance rates were calculated to result in income losses of between $\$ 10.00$ and $\$ 25.00$ per carcass for HSCW and between $\$ 7.00$ and $\$ 20.00$ per carcass for fat depth (McPhee and Walmsley, 2014). In an additional analysis of 20,000 records for long-fed feedlot cattle, $29 \%$ of carcasses were found to have missed HSCW specifications while $70 \%$ of carcasses missed the marble score specification of 3 or better (SlackSmith et al., 2009).

The BeefSpecs fat calculator was developed within the Cooperative Research Centre for Beef Genetic technologies (Beef CRC) and is a decision support tool designed to assist producers address the issue of noncompliance (Walmsley et al., 2011). BeefSpecs achieves this by combining practical information relating to animal growth and fatness, in response to changes in production environment, with the predictive powers of animal growth and compositional models (Meat Animal Research Centre Model, MARC; Keele et al., 1992; Williams and Jenkins, 1998) to predict end-point fatness. The major challenge during development of BeefSpecs was to seamlessly combine the computational power of these models with easily obtained on-farm inputs. An interpretive process was developed which relates on-farm measures to the input parameters required by growth models and in turn converts growth model outputs into easily understood animal production language (Walmsley et al., 2014). The refinements made and the predictive capacity of the underlying models allow BeefSpecs to function across a wide range of production environments while maintaining user interactions that are sufficiently simple to enable/encourage users to readily explore management options.

The focus of current carcass specifications on HSCW and carcass P8 rump fat depth (P8 Fat) addresses consumer requirements relating to portion size and external fat content but other carcass traits influence consumer perceptions of meat quality and production efficiency. The eating experience of consumers is positively influenced by intramuscular fat content or marbling (Thompson, 2004). Efficiency at the abattoir and throughout the remainder of the beef supply chain is influenced by carcass yield. Improvements in efficiency associated with higher yielding carcasses are recognised in the marketplace with premiums of $\$ 0.20 / \mathrm{kg}$ liveweight for one unit increases in muscle score across all market categories (McKiernan, 2002). These attributes have been used in combination with other production variables to predict carcass meat quality in a system known as the Meat Standards Australia Index, or MSA Index (unpublished). To continue to assist beef producers improve compliance rates and viability of their production systems, BeefSpecs needs to evolve by having the capacity to predict such traits as they are included in future carcass specifications.

The principles of using easily recorded on-farm measures as inputs for the base growth and body composition modelling systems in BeefSpecs and using producer language remain highly relevant for any future developments. Central to this is that the user interfaces for each BeefSpecs tool must remain sufficiently simple to enable/encourage end user interaction while being comprehensive enough to gather the information to run the base modelling systems. This is particularly important when expanding into new opportunities such as assisting higher level management systems such as on-farm drafting for mob formation.

This paper will describe the continued evolution of the BeefSpecs fat calculator to include predictions of lean meat yield and eating quality. Further developments of the other BeefSpecs tools will also be described.

\section{THE BEEFSPECS CALCULATOR}

\subsection{Original Development}

A dynamic steer growth model (MARC; Keele et al., 1992, Williams and Jenkins, 1998) forms the basis of the BeefSpecs fat calculator (Figure 1). The MARC model is based on the observation that an animal of a given type will, on average, have a defined body composition (fat and lean) when treated in a specified manner while growing at a particular rate. Building on the MARC model, BeefSpecs uses frame score, sex and breed type to develop a description of animal type while using initial liveweight and P8 fat to ascertain the animals initial body composition. Growth rate ( $\mathrm{kg} / \mathrm{day})$ is then used to determine final body composition after a feeding period (days). Modifications to the MARC model allow rib fat depth (mm) to be predicted 
from simulated total body fat. Rib fat is in turn used to predict P8 fat depth (mm) taking account of sex, breed type and weight of the animal (Walmsley et al., 2010). Breed type has been accommodated as an input using a dynamic visual comparison with the live animal on the user input interface (Figure 1).

The inputs used to initialise the MARC growth model include frame score (height at the hips relative to age - as a measure of animal maturity), initial P8 fatness and initial live weight. Animal sex and breed type both initialise the MARC model as well as adjust predicted body composition. The prevailing production conditions (hormonal growth promotant (HGP) treatment, and feed type [grass vs. grain]) are used in the modified MARC model to also adjust body composition. Body composition is adjusted so grain-fed animals growing at the same rate are fatter than grass-fed animals (Tudor, 1992). Growth and body composition are adjusted using a lagged response to reflect observed production responses (Oltjen et al., 1986) when animals are treated with HGPs. The prediction of body composition and subsequently P8 fat depth is driven primarily by days on feed and growth rate. Inputs relating to animal type that are located on the 'Animals tab' are demonstrated in Figure 1 along with the results section. The inputs entered into and the outputs obtained from the BeefSpecs fat calculator are group averages as opposed to individual animal measures, because of the nature of the data sets used to derive the model parameters.

\subsection{Including Lean Meat Yield and Meat Quality}

Extensions to BeefSpecs have been developed to provide users with the capacity to consider the implications their management decisions have on meat quality and production efficiency, as described by lean meat yield, in addition to focusing solely on HSCW and P8 fat. These additions have been made in anticipation of their inclusion in future carcass specifications. Both lean meat yield and MSA marble score have been incorporated into BeefSpecs with the inclusion of only one additional input, muscle score (scale 1 to 15; Figure 2). Muscle score is used in association with frame score to derive fat free mass (FFM) at maturity. In this construct, animals of the same frame score but higher muscle score are predicted to have higher lean muscle percentages and lower carcass fat percentages at maturity than lower muscle score animals. Muscle score is also used in association with $\mathrm{P} 8$ fat depth and feed type to predict carcass dressing percentage as described by McKiernan et al (2007). This dressing percentage is in turn used to calculate HSCW following McKiernan et al. (2007) with the addition of a 0.95 correction factor

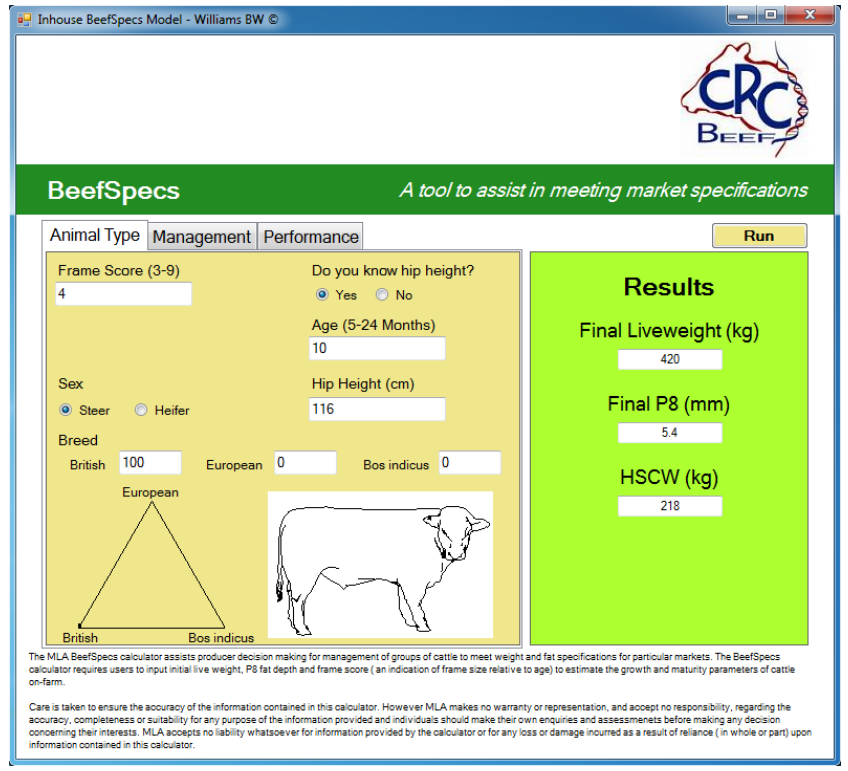

Figure 1. The user interface of BeefSpecs version 1 showing the frame score, sex and breed type inputs on the 'Animal Type' tab as well as the predicted outputs under the 'Results' heading.

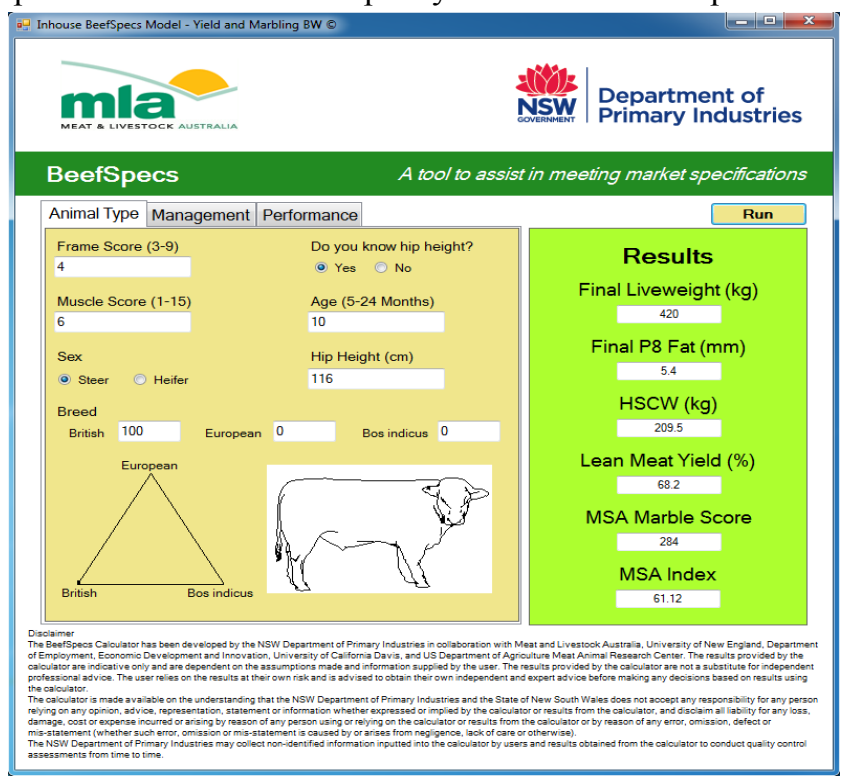

Figure 2. The user interface of BeefSpecs version 2 showing the muscle score input added to the 'Animal Type' tab and the additional outputs for lean meat yield, MSA marble score and MSA index under the 'Results' heading. 
(1) to account for carcass shrinkage between the paddock and lairage.

HSCW $=0.95 *$ Full Body Weight $*$ Dressing Percent

The addition of lean meat yield and MSA marble score builds on the pre-existing structure in BeefSpecs by partitioning empty body FFM and empty body fat mass (FM), while correcting for bone content, into carcass flesh and non-carcass flesh components. Bone content is derived from the relationship between HSCW and bone weight using data taken from Haecker (1920). Lean meat yield, shown on Figure 2, is derived as the sum of carcass FFM and the intramuscular fat (IMF) component of carcass FM relative to carcass weight (2).

Lean Meat Yield $=(($ Flesh FFM $+(0.135 *$ Flesh FM $)) /$ HSCW $) * 100$

The intramuscular fat percent is the IMF pool relative to the sum of flesh FFM and the IMF pool. The FM that is partitioned to the IMF pool was derived as $13.5 \%$ to obtain agreement between predicted IMF\% in the model and chemical IMF measured in the M. longissimus lumborum of 95 steers and heifers from the NSW DPI muscling herd progeny (2010 born). A quadratic relationship is then used to predict MSA marble score, shown on Figure 2, from the IMF\%.

The prediction of MSA marble score added to the BeefSpecs framework is used to provide a prediction of beef eating quality via linkage to the MSA index (Figure 2) using live animal measurements. In addition to the inputs and outputs provided by BeefSpecs, the MSA index also requires carcass ossification. A prediction of ossification has been developed that takes account of sex and animal live weight (Gudex et al. unpublished). This relationship was chosen amongst others tested for having variables that can be routinely recorded on-farm for use as BeefSpecs inputs and not relying on carcass trait predictions made by BeefSpecs while having comparable accuracy. To make predictions of eating quality using the MSA index, animals were assumed to not be milk-fed vealers and not sold through saleyards. The hump height input for the MSA index is set at $15 \mathrm{~cm}$ and the impact of tropical breed content is derived from the BeefSpecs Bos indicus breed input. The remaining MSA index inputs; sex, HSCW, HGP use, rib fat depth and Bos indicus breed content, are either inputs to or outputs from BeefSpecs.

\subsection{How accurate are the additions to BeefSpecs?}

The accuracy of prediction made by the additions to BeefSpecs has been evaluated using 2 datasets. Dataset 1 contained 77 Angus steers from the NSW DPI muscling herd (2011 born) that were slaughtered following pasture finishing at 19 months of age or after a 100 day feedlot finishing period at 22 months of age. The untrimmed boneless primals from these steers were scanned using a Picker Ultra Z Spiral CT scanner (Philips Medical Imaging Australia, Sydney NSW) to estimate lean and fat tissue weights. The M. longissumus lumborum was used to determine IMF\%. The additions to BeefSpecs generally produced good predictive accuracy. The lean meat weight mean bias of $-5.55 \mathrm{~kg}$, a regression slope close to one, a correlation between observed and predicted of 0.85 and the majority of the mean square error of prediction (MSEP) being in the random component suggests BeefSpecs is able to accurately predict lean meat yield (Table 1). The mean bias for MSA marble score is pleasing, given MSA marble score is recorded in 10 unit increments (e.g. 300, 310, $320 \ldots)$. The majority of MSEP being attributable to the random component is also a good result. However, the regression slope was 0.58 , the correlation between observed and predicted was 0.48 and the MSEP was relatively large. These inaccuracies could be attributable to the inaccuracies in predictions of IMF\%. The prediction of IMF\% had a high mean bias, a low regression slope, a low correlation with observed and a large proportion of MSEP in the bias component. These errors may be due to the small range in $\mathrm{IMF} \%$ in this dataset particularly given IMF\% values for the feedlot finished animals were not obtainable.
Table 1. Comparison of predicted and observed carcass traits that have been incorporated into BeefSpecs using two evaluation datasets.

\begin{tabular}{lcccc}
\hline \multirow{2}{*}{ Dataset } & \multicolumn{3}{c}{ Dataset 1 } & Dataset 2 \\
\cline { 2 - 5 } & $\begin{array}{c}\text { Lean Meat, } \\
\mathrm{kg}\end{array}$ & $\begin{array}{c}\text { MSA } \\
\text { Marble }\end{array}$ & $\begin{array}{c}\text { IMF, } \\
\%\end{array}$ & $\begin{array}{c}\text { IMF, } \\
\%\end{array}$ \\
\hline $\mathrm{n}$ & 77 & 77 & 40 & 527 \\
Mean Obs & 169.91 & 359.61 & 3.30 & 3.44 \\
Mean Pred & 175.46 & 359.80 & 2.60 & 3.35 \\
Mean Bias & -5.55 & -0.19 & 0.70 & 0.09 \\
b coefficient & 1.08 & 0.58 & 0.45 & 0.60 \\
Correlation, $\mathrm{r}$ & 0.85 & 0.48 & 0.31 & 0.64 \\
MSEP* & 245.97 & 4321.80 & 1.38 & 2.08 \\
Root-MSEP & 15.68 & 65.74 & 1.17 & 1.44 \\
Bias & 12.53 & 0.00 & 35.67 & 0.39 \\
Slope & 1.32 & 13.85 & 8.66 & 23.55 \\
Random & 86.15 & 86.15 & 55.67 & 76.06 \\
t-test p-value & 0.00 & 0.98 & 0.00 & 0.15 \\
\hline
\end{tabular}

*MSEP = mean square error of prediction; Bias = MSEP attributable to overall prediction bias; Slope $=$ MSEP attributable to deviation of regression slope from unity; random = MSEP attributable to random error. 
Dataset 2, containing 527 animals, was obtained from the Cooperative Research Centre for Cattle and Beef Quality (1993-1997) and included only IMF\% data from Bos taurus and Bos indicus animals. Predictions of IMF\% were more accurate than those in dataset 1 (Table 1), however the correlation between observed and predicted was not one nor was the regression slope which resulted in a higher than desirable portion of the MSEP being attributed to the slope component. These results suggest predictive accuracy can be improved and work is progressing to obtain additional data from a wider range of cattle to achieve these improvements.

\section{OTHER TOOLS IN THE BEEFSPECS FRAMEWORK}

The description above illustrates improvements that have been made to the BeefSpecs framework to provide users with predictions of carcass traits that are expected be included in future carcass specifications. Underpinning BeefSpecs with a research model that embodies biological and physical rules about animal growth and development allowed easy expansion to provide additional tools for addressing new opportunities (e.g. on-farm drafting and feedlot optimisation; Walmsley et al., 2011). These tools have also been developed further with only one additional input required (i.e. muscle score) and the removal of the dressing percentage input. Developments to these tools other than those described above are detailed below.

\subsection{On-Farm Drafting}

The objective of developing the BeefSpecs on-farm drafting tool was to allow users to explore dividing animals into sub-groups that may require different management strategies to better achieve specifications. This tool uses the BeefSpecs framework without changing the underlying MARC model to generate predictions of future P8 fat depth for individuals based on their current liveweight and P8 fat depths in association with the expected average growth rate and production conditions. Animals are then sorted based on expected performance against targeted market specifications to enable users to explore the predicted impacts that management changes will have on the performance of sub-groups independent of the whole group average. Developments to the drafting tool include a description of the expected variability in the predicted performance of the individuals (Figure 3) and market specifications other than HSCW and P8 fat depth used to value carcasses for one processing company (additional specifications are not shown in Figure 3). The expected variability in predicted performance is presented as a white cloud surrounding each individuals predicted $\mathrm{HSCW}$ and $\mathrm{P} 8$ fat (Figure 3). This cloud is derived from a coefficient of variation of $10 \%$ in average growth rate to represent potential minimum and maximum HSCW and a constant error of $\pm 3 \mathrm{~mm}$ for P8 fat is assumed based on evaluations conducted during the development of BeefSpecs. Presenting expected variability in predictions in this manner provides users with an indication of the potential risks of their animals not complying with specifications even though they were predicted to comply. A mechanism has also been developed that considers this variability when assessing the economic implications of applying different management strategies to

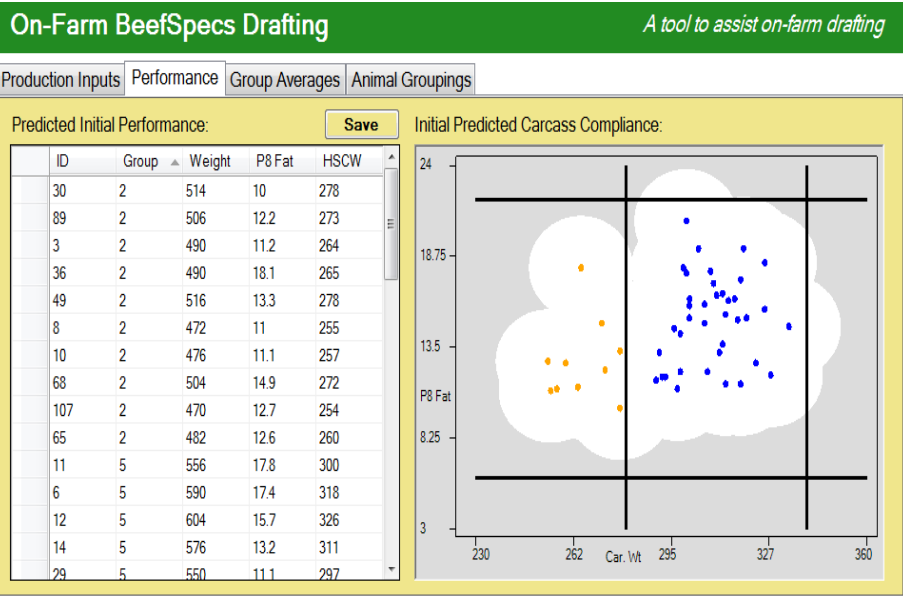

Figure 3. On-farm drafting tool showing P8 fat and hot standard carcass weight predictions for individuals (left) and a comparison to market specifications (right). Non-compliant data are coloured orange and compliant data are blue. The expected variability in predicted individual performance is shown as a white cloud. different animal groups.

\subsection{Pasture Optimisation}

Mayer et al. (2007) used HSCW and P8 fat predicted by BeefSpecs in conjunction with an optimisation engine to allocate animals to feedlot pens based on compliance with market specifications. The optimisation indicated that penning animals based on how well and when they met market specifications increased feedlot gross margin. This illustrated that BeefSpecs can be used to improve feedlot profit and led to the development of decision-rules that underpin a feedlot optimisation tool based on the BeefSpecs framework. 
This tool allocates animals to different pens based on predictions of individual P8 fat levels following nominated growth and management conditions with the aim of optimising time on feed required to reach target market specifications and thereby minimizing production costs while maximising profit. The principles developed from Mayer et al. (2007) and contained in the feedlot optimisation tool have been applied in a pasture-based tool to allocate animals to mobs with a similar propensity to meet market specifications. The major development in this process has been the derivation of new between-animal variability estimates from which the stochastic price discounts grid is constructed (Mayer et al., 2013). The standard deviations used in the pasture-based optimisation are $25.4 \mathrm{~kg}$ and $3.59 \mathrm{~mm}$ compared to $23 \mathrm{~kg}$ and $5.1 \mathrm{~mm}$ used in the feedlot optimisation. The optimisation tools functionality has been increased by developing the capacity to readily change the carcass pricing schedule which is used to derive the stochastic price discounts grid (Figure 4).
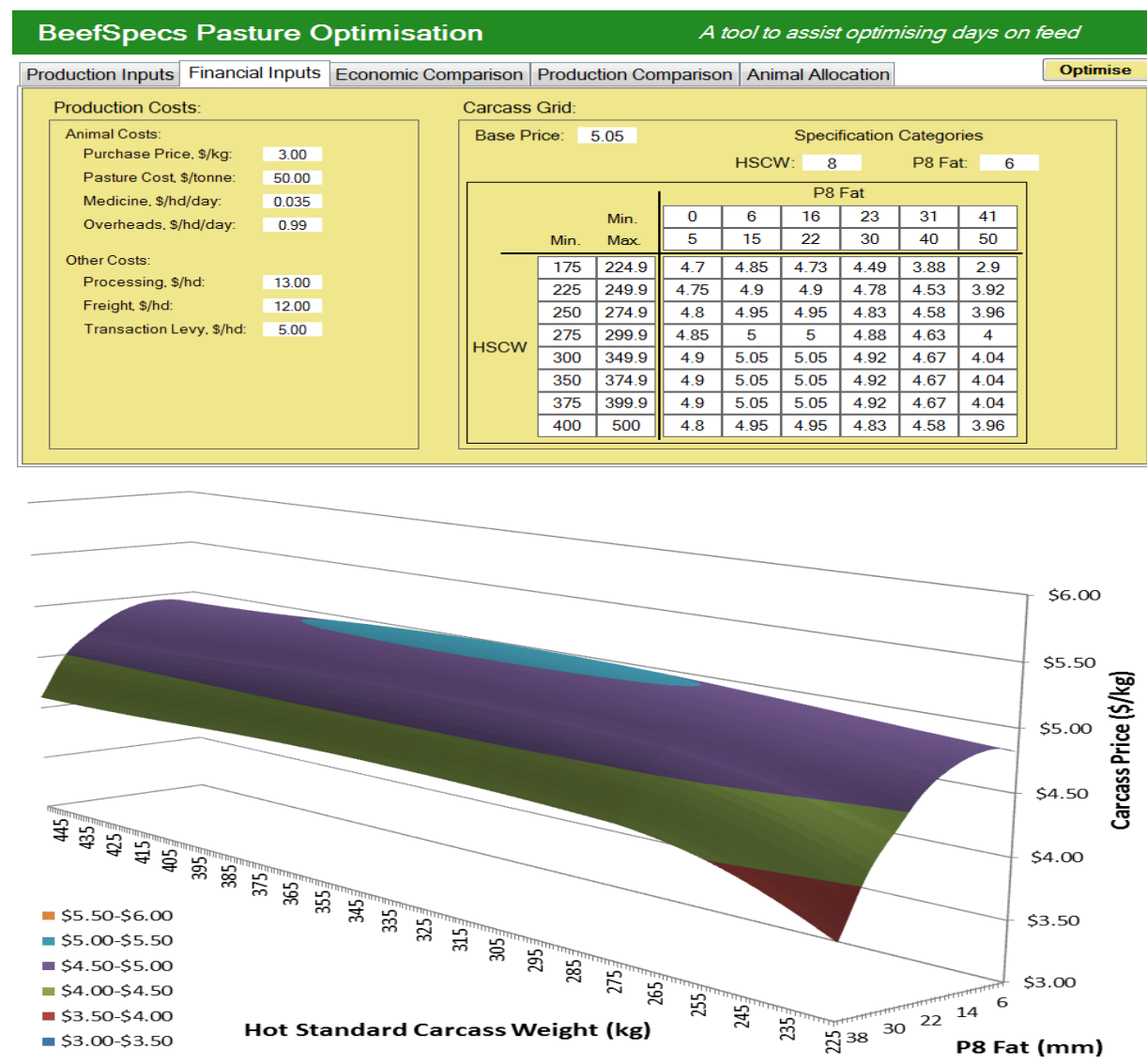

Figure 4. The capacity to readily change carcass pricing schedules (above) has been developed to add functionality to the pasture optimisation tool. The stochastic price discount grid (below) is shown for the pasture finished carcass specifications from one pasture based market entered above.

\subsection{Future Developments}

A study into potential sources of error in BeefSpecs inputs (McPhee and Walmsley, 2014) demonstrated that the accuracy of BeefSpecs predictions is particularly sensitive to the accuracy of live animal assessments for frame score and P8 fat. Research has been conducted to examine the feasibility of using 3D images reconstructed from RGBD cameras to predict these live animal traits (McPhee, 2015) as a way of improving the accuracy of prediction by reducing user error in live animal assessment. Efforts are also being made to link these image systems with BeefSpecs via a trading pipeline to help facilitate the development of valuebased trading mechanisms rather than following traditional commodity trading systems.

\section{CONCLUSIONS}

The BeefSpecs framework combines the knowledge obtained from growth path experiments with that contained in animal growth and body composition models, to assist beef producers make management decisions that enable their cattle to better meet market specifications. Developments have been undertaken to extend the predictive capabilities of the BeefSpecs framework to include beef eating quality and production 
efficiency as described by lean meat yield. The predictive accuracy of these developments has generally been good given the limited data available for development and evaluation. The development of additions to the BeefSpecs on-farm drafting tool and extension of the feedlot optimisation tool to pasture-based production systems has been described. Further development and implementation of these tools is expected to occur in the future as methods to readily record BeefSpecs inputs on-farm and hard-to-measure traits in abattoirs become commercially viable. Routinely recording hard-to-measure traits in abattoirs will also facilitate the inclusion of these traits in carcass specifications, in particular lean meat yield.

\section{ACKNOWLEDGMENTS}

The authors gratefully acknowledge the in-kind and funding support from NSW Department of Primary Industries and the financial support of Meat and Livestock Australia. The assistance of commercial abattoir operations during collection of data has been invaluable in the continued development of BeefSpecs.

\section{REFERENCES}

Haecker T.L. (1920). Investigations in beef production. Agricultural experiment station research bulletin. University of Minnesota, St. Paul, Minnesota. 110.

Keele J.W., Williams C.B., and Bennett G.L. (1992). A computer model to predict the effects of level of nutrition on composition of empty body gain in beef cattle: I. Theory and development. Journal of Animal Science, 70, 841-857.

Mayer D.G., McPhee M.J., Oddy V.H., Kinghorn B.P., Oltjen J.W., McKiernan W.A., and Wilkins J.F. (2007). Evolutionary computation targeting market fat specifications in beef steers. In: 'MODSIM 2007 International Congress on Modelling and Simulation.' (L. Oxley and D. Kulasiri eds.). Modelling and Simulation Society of Australia and New Zealand. University of Canterbury, Christchurch, New Zealand. p. 81-87.

Mayer D.G., Walmsley B.J., McPhee M.J., Oddy V.H., Wilkins J.F., Kinghorn B.P., Dobos R.C. and McKiernan W.A. (2013). Integrating stochasticity into the objective function avoids Monte Carlo computation in the optimisation of beef feedlots. Computers and Electronics in Agriculture, 91, 30-34.

McKiernan W.A. (2002). Importance of various cattle traits in the national livestock reporting system. Animal Production in Australia, 24, 329.

McKiernan W.A., Gaden B. and Sunstrom B. (2007). Dressing percentages for cattle. Primefact 340, NSW Department of Primary Industries. 3.

McPhee M.J. and Walmsley B.J. (2014). Assessment of compliance in grass-fed cattle and evaluation of increasing accuracy of BeefSpecs inputs. Final report. Meat and Livestock Australia, North Sydney. 46.

McPhee M.J. (2015). Trait measurement from 3D image. Final report. Meat and Livestock Australia, North Sydney. 36.

Oltjen J.W., Bywater A.C., Baldwin R.L. and Garrett W.N. (1986) Development of a dynamic model of beef cattle growth and composition. Journal of Animal Science, 62, 86-97.

Slack-Smith A.R., Griffith G.R., and Thompson J.M. (2009). The cost of non-compliance to beef market specifications. Australasian Agribusiness Review, 17, 178-190.

Thompson J.M. (2004). The effects of marbling on flavour and juiciness scores of cooked beef, after adjusting to a constant tenderness. Australian Journal of Experimental Agriculture, 44, 645-652.

Tudor G.D. (1992). Effect of diet on fat deposition in cattle. In: 'Proceedings of the Australian Society of Animal Production.' No. 19. La Trobe University, Melbourne. p. 89.

Walmsley B.J., Oddy V.H., McPhee M.J., Mayer D.G. and McKiernan W.A. (2011). Development of the BeefSpecs fat calculator: A tool designed to assist decision making to increase on-farm profitability. In: 'MODSIM 2011 International Congress on Modelling and Simulation.' (F. Chan, D. Marinova and R.S. Anderssen eds). Modelling and Simulation Society of Australia and New Zealand. Perth Convention and Exhibition Centre, Perth, Australia. p. 898-904.

Walmsley B.J., Wolcott M.L., and McPhee M.J. (2010). Modelling the relationship between scanned rump and 12th-rib fat in young temperate and tropical bovines: Model development and evaluation. Journal of Animal Science, 88, 1848-1859.

Walmsley B.J., McPhee M.J., and Oddy V.H. (2014). Development of the BeefSpecs fat calculator to assist decision making to increase compliance rates with beef carcass specifications. Animal Production Science, 54, 2003-2010.

Williams C.B., and Jenkins T.G. (1998). A computer model to predict composition of empty body weight changes in cattle at all stages of maturity. Journal of Animal Science, 76, 980-987. 